\title{
Study of Pre-disposing Factors of Acute Exacerbation of Chronic Obstructive Pulmonary Disease and Antibiotic Prescribing Pattern with Reference to Antibiotic Sensitivity Test.
}

\section{Shrestha $R,{ }^{1,2}$ Shrestha $B,{ }^{1}$ Shakya Shrestha $S,{ }^{1,2}$ Pant $A,{ }^{1}$ Prajapati $B,{ }^{3}$ Karmacharya BM ${ }^{4}$}

${ }^{1}$ Department of Pharmacology

School of Medical Sciences, Kathmandu University

${ }^{2}$ Research \& Development Division

Dhulikhel Hospital, Kathmandu University Hospital

${ }^{3}$ Department of Internal Medicine

Dhulikhel Hospital, Kathmandu University Hospital

${ }^{4}$ Department of Community Program

Dhulikhel Hospital, Kathmandu University Hospital

\section{Corresponding Author}

Rajeev Shrestha

Department of Pharmacology

Dhulikhel Hospital, Kathmandu University Hospital

School of Medical Sciences

Dhulikhel, Kavre, Nepal.

E-mail: rmaleku@hotmail.com

Citation

Shrestha R, Shrestha B, Shrestha SS, Pant A, Prajapat B, Karmacharya BM. Study of Pre-disposing Factors of Acute Exacerbation of Chronic Obstructive Pulmonary Disease and Antibiotic Prescribing Pattern with Reference to Antibiotic Sensitivity Test. Kathmandu Univ Med J 2015;51(3):250-5.

\section{ABSTRACT}

\section{Background}

Chronic Obstructive Pulmonary Disease (COPD) affects about 329 million people worldwide, which is nearly $5 \%$ of the entire global population. In the context of Nepal, COPD accounts for $43 \%$ of the non-communicable disease burden and $2.56 \%$ of hospitalizations. Various pre-disposing factors like bacterial, viral, fungal, smoking, occupational exposures and genetic factors have been proposed to precipitate COPD and its exacerbation though, the definitive pre-disposing factors and factors related to acute exacerbation have not been determined in the context of Nepal.

\section{Objective}

To find out the pre-disposing factors and the related causative agents for COPD.

\section{Method}

A cross sectional study was conducted in a tertiary care hospital. Patients of all age group who were diagnosed as COPD and admitted in the hospital were included in this study. Patients were interviewed using structured questionnaire. The sociodemographic data including personal and medical history were recorded from those participants. In addition, sputum from those patients was sent for culture to investigate the possible responsible pathogens as well as its antibiotic sensitivity pattern.

\section{Result}

A total of 150 patients having Acute Exacerbation of Chronic Obstructive Pulmonary Disease (AECOPD) who have admitted from either emergency or out-patient department of the hospital were included in this study. Among the total number of patients, more than half of them were female $(n=82)$. In addition, analysis of occupations shows that most of them were either farmer $(36.0 \%)$ or housewife $(30.7 \%)$. In total studied patients $(n=150)$, most of them were using traditional firewood (83\%) for cooking purpose and majority of patients (91\%) were smokers. Most of the sputum samples show growth of gram-positive cocci (26.7\%) and gram negative bacilli (27.5\%). Considering the overall sensitivity pattern, the higher sensitivity was recorded for Co-trimoxazole and Ciprofloxacin while higher rate of resistance was noted for Penicillin group of drugs. The most widely used antibiotics were found to be Cephalosporin group of drugs (68\%).

\section{Conclusion}

The present study revealed that the case of COPD is more in female and the commonest pre-disposing factor is found to be smoke/firewood. Cephalosporin group of drugs is the most commonly prescribed drug.

\section{KEY WORDS}

$A E C O P D$, firewood, pre-disposing factor and therapy of COPD 


\section{INTRODUCTION}

Chronic Obstructive Pulmonary Disease (COPD) affects about 329 million people worldwide, which is nearly $5 \%$ of the entire global population. In 2011, it was ranked as the fourth-leading cause of death, killing over three million people. ${ }^{1}$ According to $\mathrm{WHO}$, the total number of deaths in the world from COPD is projected to increase by more than $30 \%$ in the next 10 years and by 2030 , COPD would become the fourth leading cause of death worldwide. In the context of Nepal, COPD accounts for $43 \%$ of the non-communicable disease burden and $2.56 \%$ of hospitalizations. ${ }^{2}$ Various pre-disposing factors like bacterial, viral, fungal, smoking, occupational exposures and genetic factors have been proposed to precipitate COPD and its exacerbation though, the definitive pre-disposing factors and factors related to acute exacerbation have not been determined in the context of Nepal. ${ }^{2-4}$ In addition, various antibiotics have been prescribed in the therapy of Acute Exacerbation of Chronic Obstructive Pulmonary Disease (AECOPD) but being used without any antibiotic susceptibility test which also might delay in recovery.

Therefore, in attempt of establishing the pre-disposing factors, related causative agents and the management of COPD with reference to antibiotic susceptibility test, this study was conducted in the tertiary care hospital.

\section{METHODS}

A cross sectional study was conducted (February 2014 to January 2015) in a tertiary care hospital at Dhulikhel, Kavre after obtaining the ethical approval from the Institutional Review Committee, Kathmandu University School of Medical Sciences. Patients of all age group who were diagnosed as COPD and admitted in the hospital were included in this study. However, those patients who were not admitted in the hospital and who do not want to give consent to participate in this study were excluded.

Patients who met the inclusion criteria's were informed about the study and written consent was obtained from them to include their data for the research purpose. Patients were interviewed using structured questionnaire. The socio-demographic data including personal and medical history were recorded from those participants. In addition, sputum from those patients was sent for culture to investigate the possible responsible pathogens as well as its antibiotic sensitivity pattern. During antibiotic sensitivity test, antibiotic discs were selected on the basis of commonly used antibiotics in clinical practice.

The collected data entered in a separate spreadsheet using EXCEL 2007 and using spss 17.0, frequency and percentage tables were generated in relevant tables and charts.

\section{RESULTS}

\section{Demographic characteristics of study population}

A total of 150 patients having AECOPD who have admitted from either emergency or out-patient department of the hospital were included in this study. Among the total number of patients, more than half of them were female $(n=82)$. Analyzing the age of patients, it was found that maximum number of patients $(76.0 \%)$ were the age between $60-80$ years who were admitted with AECOPD (Table 1). The present study has also revealed that most of patients (81.3\%) were illiterate of which more than half of them were female (54.7\%). In addition, analysis of occupations shows that most of them were either farmer (36.0\%) or housewife (30.7\%; Table 1$)$.

Table 1. Demographic characteristics of study population

\begin{tabular}{|lc|}
\hline $\begin{array}{l}\text { Personal History }(\mathbf{n = 1 5 0 )} \\
\text { Gender }\end{array}$ & No. of Patients (\%) \\
\hline Male & $68(45.3)$ \\
\hline Female & $82(54.7)$ \\
\hline Age & \\
\hline$<60$ & $23(15.3)$ \\
\hline $60-80$ & $114(76.0)$ \\
\hline$>80$ & $13(8.7)$ \\
\hline Literacy & \\
\hline Illiterate & $122(81.3)$ \\
\hline Literate & $28(18.7)$ \\
\hline Occupation & \\
\hline Agriculture & $54(36.0)$ \\
\hline House-wife & $46(30.7)$ \\
\hline Business & $26(17.3)$ \\
\hline Service & $6(4.0)$ \\
\hline Others* & $18(12.0)$ \\
\hline * Army, Police, teachers & \\
\hline
\end{tabular}

\section{Pre disposing risk factors and personal history}

The present study has analyzed the pre disposing risk factors like traditional fire wood use, smoking and seasonal effect including alcohol consumption pattern among COPD patients. In total studied patients $(n=150)$, most of them were using traditional firewood (83\%) for cooking purpose and majority of patients (91\%) were smokers (Table 2). Among smokers, more than half of them (60\%) smoke at least 5 to 10 times a day and nearly $43 \%$ of smokers were smoking 5-10 stick of cigarettes per day. In addition, the present study has also revealed that $53 \%$ of COPD patients had smoked at least 20 years. Among those COPD patients, nearly $50 \%$ of them were hospitalized 5 to 10 times since their diagnosis and $44 \%$ of them suffered respiratory problem mostly during winter season. Most of them (86\%) reported a cough with sputum during morning and night. 
Table 2. Various pre-disposing factors and personal history of COPD patients.

\begin{tabular}{|c|c|}
\hline Patients History & No. of Patients (\%) \\
\hline \multicolumn{2}{|l|}{ Smoking History $(n=150)$} \\
\hline Yes & $15(10.0)$ \\
\hline No & $135(90.0)$ \\
\hline \multicolumn{2}{|c|}{ Frequency of Smoking per Day ( $n=135)$} \\
\hline$<5$ & $37(27.4)$ \\
\hline $5-10$ & $90(66.6)$ \\
\hline$>10$ & $8(6.0)$ \\
\hline \multicolumn{2}{|c|}{ Cigarette sticks per Day ( $n=135$ ) } \\
\hline$<5$ & $8(6.0)$ \\
\hline $5-10$ & $64(47.4)$ \\
\hline$>10$ & $63(46.6)$ \\
\hline \multicolumn{2}{|c|}{ Duration of Smoking (yrs) ( $n=135)$} \\
\hline$<20$ yrs & $12(8.9)$ \\
\hline $20-40$ yrs & $79(58.6)$ \\
\hline $40-60$ yrs & $43(31.8)$ \\
\hline$>60 \mathrm{yrs}$ & $1(0.1)$ \\
\hline \multicolumn{2}{|c|}{ Spouse Smoking History $(n=150)$} \\
\hline No & $47(31.3)$ \\
\hline Yes & $103(68.7)$ \\
\hline \multicolumn{2}{|l|}{ Cooking Method $(n=150)$} \\
\hline Traditional Firewood & $125(83.3)$ \\
\hline LPG Gas & $3(2.0)$ \\
\hline Fireless Cooking & $22(14.7)$ \\
\hline \multicolumn{2}{|c|}{ Frequency of hospitalization $(n=150)$} \\
\hline$<5$ times & $57(38.0)$ \\
\hline 5-10 times & $74(49.3)$ \\
\hline$>10$ times & $19(12.7)$ \\
\hline \multicolumn{2}{|l|}{ Difficult Season $(n=150)$} \\
\hline Winter & $65(43.3)$ \\
\hline Summer & $16(10.7)$ \\
\hline All Season & $69(46.0)$ \\
\hline
\end{tabular}

Microorganism growth in sputum culture and sensitivity pattern

\section{Isolation of microorganism from sputum culture}

Among 150 patients, sputum samples collected from 127 patients were subjected for microbiological test and the rest of sputum samples were discarded due to either insufficient sputum or only saliva content.

The present data show that there was no growth in about $32.2 \%$ sputum samples whereas rest of the samples has shown growth of bacteria and fungi (Table 3). Most of the sputum samples show growth of gram positive cocci (26.7\%) and gram negative bacilli (27.5\%). Some sputum samples also have fungal growth $(n=4)$.

\section{Isolation of bacteria during culture of sputum}

Out of 127 patients whose sputum samples were taken for
Table 3. Growth of microorganisms in sputum culture.

\begin{tabular}{|l|l|}
\hline Type of Growth $(\mathbf{n = 1 2 7})$ & No. of Patients (\%) \\
\hline No Growth & $41(32.2)$ \\
\hline Gram positive cocci & $34(26.7)$ \\
\hline Gram negative cocci & $2(1.6)$ \\
\hline Gram positive bacilli & $11(8.7)$ \\
\hline Gram negative bacilli & $35(27.5)$ \\
\hline Fungal hyphae & $4(3.1)$ \\
\hline
\end{tabular}

microbiological evaluation, only 87 of them has shown the growth of microorganisms. According to the given data, most samples presented the growth of normal flora (43\%) and rest of them had pathogenic microorganisms $(n=50)$. Out of 50 sputum samples that depicted the growth of pathogenic microorganisms, most were Escherichia coli (14.0\%), Streptococcus pneumonia (11.6\%) and Klebsiella pneumonia (10.4\%; Table 4). Some samples also showed the growth of a fungus i.e. Aspergillus fumigates (5\%).

Table 4. Types of microorganism isolates in sputum culture.

\begin{tabular}{|ll|}
\hline Types of Microorganism $(\mathbf{n = 8 6})$ & No. of Patients (\%) \\
\hline Normal flora & $36(42.0)$ \\
\hline Klebsiella pneumoniae & $9(10.4)$ \\
\hline Stretococcus pneumoniae & $10(11.6)$ \\
\hline Escherichia coli & $12(14.0)$ \\
\hline Aspergillus fumigate & $4(4.7)$ \\
\hline Acinetobacter spp. & $7(8.1)$ \\
\hline Pseudomonas aeruginosa & $6(7.0)$ \\
\hline Enterobacter spp. & $1(1.1)$ \\
\hline Klebsiella oxytoca & $1(1.1)$
\end{tabular}

\section{Antibiotic susceptibility testing}

The susceptibility testing was done by disc diffusion method. The antibiotics used were Co-trimoxazole, Ciprofloxacin, Cephalosporins (Ceftriaxone, Cefixime and Ceftazidime), Penicillins (Amoxycillin and Ampicillin), Macrolides (Azithromycin and Erythromycin) and Aminoglycosides (Amikacin and Gentamicin). Considering the overall sensitivity pattern, the higher sensitivity was recorded for Co-trimoxazole and Ciprofloxacin while higher rate of resistance was noted for Penicillin group of drugs.

In terms of antibiotics sensitivity, the present study shows that Ciprofloxacin (75\%) seemed to be highly effective to Escherichia coli, which is followed by Azithromycin (33\%), Aminoglycosides; Gentamicin (33\%), Amikacin (25\%) and Co-trimoxazole (25\%). However, Escherichia coli was resistant against Penicillin group of drugs including Amoxicillin (75\%) and Ampicillin (67\%). Similarly, Streptococcus pneumoniae showed higher sensitivity to Co-trimoxazole (91\%), Amoxicillin (91\%), Ceftriaxone (82\%) and average sensitivity to Erythromycin (55\%) and Ampicillin (55\%). However, the minimal effect was observed in Cefixime (36\%) and Amikacin (9\%). The present study has 
revealed that Co-trimoxazole (100\%), Ciprofloxacin (100\%) and Amikacin (88\%) were highly effective against Klebsiella pneumonia whereas these microorganisms were resistant to penicillins (63\%).

Further, Acinetobacter spp were sensitive towards amikacin (86\%), Ciprofloxacin (86\%) and Co-trimoxazole (75\%) while Ampicillin (71\%), Amoxicillin (29\%), Cotrimoxazole (14\%) and Ceftazidime (29\%) were resistant. Pseudomonas aeruginosa seemed sensitive towards Ciprofloxacin (83\%) and Amikacin (83\%) while less sensitive to Gentamycin (33\%), Ceftriaxone (17\%) and Azithromycin (33\%). Furthermore, Ciprofloxacin (100\%) and Cefixime $(100 \%)$ were highly effective against entero-bacter whereas Amoxicillin showed $100 \%$ resistant. Similarly, Cotrimoxazole and Ciprofloxacin were highly effective against Klebsiella oxytoca whereas, it shows complete resistant to penicillin.

\section{Antibiotics use pattern}

In addition to drugs used for the management of COPD, antibiotics have been prescribed to almost all the patients. Some patients were in single antibiotic therapy while others with two antibiotics at a time. The most widely used antibiotics were found to be Cephalosporin group of drugs (68\%) which was followed by Macrolide, Azithromycin (32\%) and penicillin group (23\%; figure 1). Few patients were also prescribed with Levofloxacin $(n=4)$. Moreover, patients were prescribed two antibiotics at the same time. The widely prescribed antibiotic combination was one group from Cephalosporin group plus Azithromycin (22\%). About $3 \%$ of the treated patients were also prescribed with one group from Cephalosporin plus Amoxicillin+Clavulanate.

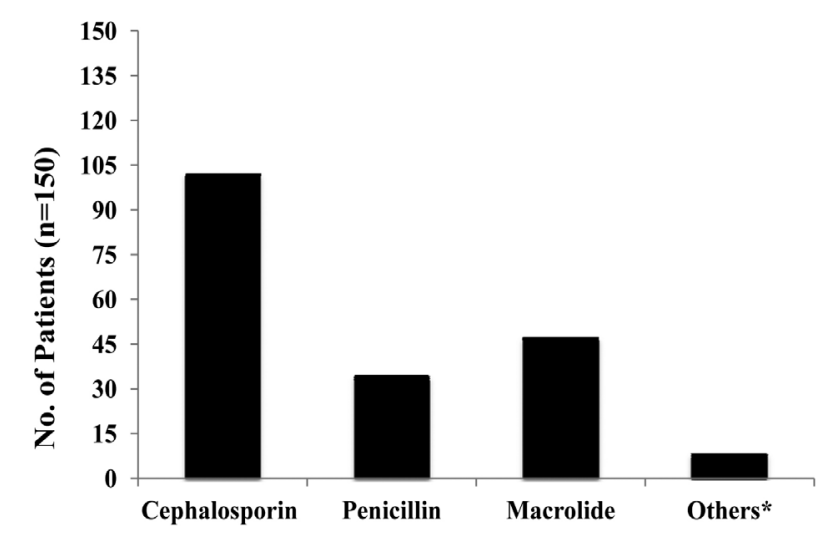

*Levofloxacin, Piperacillin+Tazobactam (not included in Penicillin group)

Figure 1. Bar chart showing antibiotics use pattern in COPD patients.

\section{DISCUSSION}

Among the studied population, majority of patients diagnosed with COPD were females. The higher number of female COPD patients in our study might be due to the fact that they have higher exposure to biomass fuels and frequent smoking history. ${ }^{5}$ This can be co-related with the study, which depicted the extensive use of biomass fuels by female individuals for long period of time increased the risk of indoor air pollution. ${ }^{6}$ Several other studies presented similar findings where females are predominantly affected. ${ }^{6-9}$ According to the $\mathrm{WHO}$, previously COPD was more common in men while because of increased tobacco use among females in high- income countries and the extensive exposure to indoor biomass fuels used for cooking and heating in low-income countries, both genders are almost equally affected by the disease. ${ }^{10}$ However, some studies showed the higher prevalence of male suffers than the women because of increased frequency of smoking among men. ${ }^{11}$

The present study showed the frequency of COPD sufferers increased gradually with age and leveled off at the age group of $60-80$ years after which the predominance decreased sharply which can be correlated with the study where smoking cigarette for a prolonged period of time might deteriorate the mucosal lining of the respiratory tract causing obstruction in breathing and lead to the disease. ${ }^{12}$ The decreasing trend of COPD patients after the age of 80 years might be due to the average life expectancy of people in Nepal is 68.2 years. ${ }^{13}$

Further, among the studied population, majority of patients were involved in occupations like agriculture and household works. Farmers seemed more exposed to inorganic dust and soil during agricultural works, which may cause chronic obstruction of lung airflow that might interfere with normal breathing. ${ }^{14}$ Exposure to dusts remained one of the risk factors of COPD where one of the studies showed prolonged exposure to dusts particles would lead to obstruction in breathing. ${ }^{4} \mathrm{~A}$ previous study has reported 1,085 deaths from COPD in workers with occupational exposure to vapors, gases, fumes, and dust. ${ }^{15}$ Therefore, it is suggested that higher exposure to such hazards increases the mortality in COPD patients. ${ }^{15,16}$ Similarly, in household works, inefficient cooking fuels and technologies produce high levels of household air pollution with a range of health-damaging pollutants, including small particles that penetrate deep into the lungs. ${ }^{17}$ In consistent with our results, one of the studies illustrated greater exposures among women, who spend the most times near the domestic firewood. ${ }^{17,18}$

Furthermore, in the present study, majority of COPD patients were smokers. This finding correlates with a study, which showed smokers in Nepal, have around $70 \%$ greater chance of developing COPD, ${ }^{2}$ as smoking remained one of the important risk factors for developing COPD. ${ }^{19}$ Further, in our study, the greater frequency of female patients smoked cigarettes. This finding is supported by one of the previous study, which showed that women are more susceptible to the harmful effects of smoke than men. ${ }^{17}$ In addition, those women who smoke cigarette during pregnancy may 
increase the risk of transmitting COPD in their child as well. ${ }^{20}$

Interestingly, the present study revealed that only $9 \%$ of COPD patients were non-smokers. Our findings were consistent with some previous studies, which showed that few patients who developed COPD never smoked. ${ }^{15,21}$ Moreover, it has been shown that the incidence of mortality was higher in COPD sufferers who were either non-smokers or passive smokers. ${ }^{22}$ It is suggested that the prolonged exposure to traditional firewood and air pollution such as inorganic dusts might deteriorate the mucosal lining of respiratory tract at a faster rate than the patients who smoke cigarettes. ${ }^{22}$

In an attempt to identify the isolates in sputum culture of COPD patients, the present study has shown predominantly gram-negative bacilli during gram staining, which was followed by gram-negative cocci. This result is correlated with the previous studies, which has shown that gram-negative bacilli outnumbered the growth of other microorganisms in COPD patients. ${ }^{23,24}$ This finding has been interperated with the rampant use of antibiotics in patients with severe airflow obstruction resulting in resistance development by gram-negative bacilli. ${ }^{25}$

In the present study, the maximum growth was found to be normal flora (42\%). This high growth of normal flora can't rule out viral cause as the identification of viral origin pathology is beyond the scope of the present study. The further study can be conducted to identify the viral cause. Among pathogenic growth, the most common bacteria isolated were found to be Escherichia coli (14\%), followed by Streptococcus pneumonia (11.6\%). However, other studies have shown different types of pathogens distribution. ${ }^{12,26}$ Some study has shown the highest growth of Streptococcus pneumonia, ${ }^{26}$ while other study has shown was Klebsiella pneumonia. ${ }^{12}$ This various results from different study shows a geographical variation of pathogens and strongly suggests that the use of antibiotics should be based on the antibiotic sensitivity test or on the basis of its antibiotic guidelines. Interestingly, in the present study, one case of Klebsiella oxytoca was found. It is rare species to grow in sputum of patients therefore, further study can be conducted focusing on this pathogen.

The present study has shown that the COPD patients were prescribed with broad-spectrum antibiotics. The antibiotics commonly prescribed empirically for lower respiratory tract infections and several studies have proposed that the antibiotic treatment of acute bacterial exacerbations was the foundation of medical treatment. ${ }^{25,27}$ However, the role and choice of antibiotics in the treatment of COPD exacerbations is still a debate and recommendations for antibiotics use among published guidelines are often inconsistent. $^{20}$

In the present study, the higher sensitivity was recorded for Co-trimoxazole and Ciprofloxacin in antibiotic sensitivity test. The higher sensitivity with Co-trimoxazole can be correlated to lesser prescription practice of this drug, which might have led to less resistant. On the other hand, fluoroquinolones group of drugs have been suggested to be more effective against various gram-negative pathogens, ${ }^{26}$ which is also supported by the antibiotic sensitivity tests. In support of this study, quinolone group of drugs have shown effective response against majority of pathogens, ${ }^{12}$ however, it still couldn't be considered as the first option for starting treatment, as it can lead to emergence of resistant strains. ${ }^{12}$ Moreover, a study has reported that susceptibility of fluoroquinolones to microorganisms is decreasing because of its frequent prescription as antibiotics in patients with COPD to prevent exacerbation. ${ }^{26,28}$ Furthermore, this study has revealed that most microorganisms were resistant to Aminoglycosides and Penicillin group of drugs. Similar findings were shown where Penicillin was the highly resistant pathogen. ${ }^{23,25}$ This finding might be due to the rampant use of these drugs and needed to be explored focusing on the use of antibiotics in the exacerbation of COPD.

Moreover, this study was highlighted various pre disposing factors, related causative agents and also antibiotic use pattern. In addition, the present study recommends for the various multi-centered study to investigate causative agents causing AECOPD including their antibiotic sensitivity pattern which can be different according to the geographical locations.

\section{CONCLUSION}

The present study has tried to shed light on the distribution of COPD patients, which revealed that the case of COPD is more in female than male. Our study shows Escherichia coli and Streptococcus pneumoniae are the most common pathogens isolated from the sputum of COPD patients. Moreover, this study has revealed that Co-trimoxazole and Ciprofloxacin are the effective drugs against most of the pathogenic bacteria though, Cephalosporin group of drugs are commonly prescribed drugs in the therapy of Acute Exacerbation of Chronic Obstructive Pulmonary Disease (AECOPD). In conclusion, our study suggests that antibiotics needs to be prescribed according to antibiotics guidelines prepared on the basis of their antibiotic sensitivity test in the management of AECOPD. 


\section{REFERENCES}

1. WHO The 10 Leading Causes of Death in the World. World Health Organization, Fact Sheet No. 310 2011. (http://www.who.int/ mediacentre/ factsheets/ fs310_2008.pdf) [Accessed on $12^{\text {th }}$ September, 2014]

2. NHRC Prevalence of Non Communicable Disease in Nepal: Hospital Based Study. Nepal Health Research Council, vii, 1-65;2010.

3. Sethi S, Murphy T. Infection in the pathogenesis and course of chronic obstructive pulmonary disease. N Engl J Med. 2008;2355-2565.

4. Pirozzi $\mathrm{C}$ andScholand MB. Smoking cessation and environmental hygiene. Med Clin North Am. 2012;96(4):849-67.

5. Winrock International Nepal. Household energy, indoor air pollution and health impacts: status report for Nepal. [Accessed February 23, 2012]. Available from: http://pdf.usaid.gov/pdf_docs/PNADO853. pdf.

6. Bhandari $\mathrm{R}$ and Sharma R. Epidemiology of chronic obstructive pulmonary disease: a descriptive study in the mid western region of Nepal. Int J Chron Obstruct Pulmon Dis. 2012;7:253-7.

7. Mannino DM. Chronic obstructive pulmonary disease: definition and epidemiology. Respir Care. 2003;48(12):1185-91

8. Chapman KR. Chronic obstructive pulmonary disease: are women more susceptible than men? Clin Chest Med. 2004;25(2):331-41.

9. Alberta Medical Association. Guideline for The Management of Acute Exacerbations of Chronic Obstructive Pulmonary Disease (AECOPD)-2006 update (Retrieved on $6^{\text {th }}$ September 2015); http:// www.topalbertadoctors.org/download/377/AECOPD_guideline. pdf?_20150827095044.

10. WHO. Adherence to long-term therapies: evidence for action. Geneva, Switzerland: World Health Organization; 2003. Available at: http://www.who.int/chp/knowledge/publications/adherence_full_ report.pdf. (Accessed on June 12, 2014)

11. Loganathan RS, Stover DE, Shi W, Venkatraman E. Prevalence of COPD in women compared to men around the time of diagnosis of primary lung cancer. Chest. 2006;129(5):1305-12.

12. Madhavi S, Rama Rao MV, Rao RJ. Bacterial etiology of acute exacerbations of chronic obstructive pulmonary disease. J Microbiol Biotech Res. 2012; 2(3):440-4.

13. World Bank. World Development Indicators. Washington DC: World Bank; 2011.

14. Respiratory Health Hazards in Agriculture, American Journal of Respiratory and Critical Care Medicine.1998;158:S1-S76. doi: 10.1164/ajrccm.158.supplement_1. rccm1585s1

15. Torén $\mathrm{K}$ and Järvholm B. Effect of occupational exposure to vapors, gases, dusts, and fumes on COPD mortality risk among Swedish construction workers: a longitudinal cohort study. Chest 2014;145(5):992-7. doi: 10.1378/chest.13-1429.
16. Andersen ZJ, Hvidberg M, Jensen SS, Ketzel M, Loft S, Sørensen M, Tjønneland A, Overvad K, and Raaschou-Nielsen O. Chronic Obstructive Pulmonary Disease and Long-Term Exposure to Trafficrelated Air Pollution. American Journal of Respiratory and Critical Care Medicine. 2011;183(4): 455-61.

17. Sood A. Indoor fuel exposure and the lung in both developing and developed countries: An update. Clin Chest Med. 2012;33(4):649-65.

18. Kurmi OP, Lam KB, Ayres JG. Indoor air pollution and the lung in low and medium income countries. The European respiratory journal: official journal of the European Society for Clinical Respiratory Physiology. 2012. [PubMed]

19. Thorington P, Rios M, Avila G, Henry J, Haynes C, Pinto Pereira LM and Seemungal T. Prevalence of chronic obstructive pulmonary disease among stable chronic disease subjects in primary care in Trinidad, West Indies. J Thorac Dis. 2011;3(3):177-182.

20. Edgewater NJ. Global Initiative for Chronic Obstructive Lung Disease. Global Strategy for the Diagnosis, Management and Prevention of Chronic Obstructive Lung Disease. MCR Vision Inc; 2006.

21. Lamprecht B, McBurnie MA, Vollmer WM, Gudmundsson G, Welte T, Nizankowska-Mogilnicka E, et al. BOLD Collaborative Research Group) COPD in never smokers: results from the population-based burden of obstructive lung disease study. Chest. 2011;139(4):752-63.

22. Rennard S, Thomashow B, Crapo J, Yawn B, Mclvor A, Cerreta S, et al. Introducing the COPD Foundation Guide for Diagnosis and Management of COPD, recommendations of the COPD Foundation. COPD. 2013;10(3):378-89.

23. Elkorshy RI, El-Sherief RH. Gram Negative Organisms as a cause of accute exacerbation of COPD. Egyptian Journal of Chest Diseases and Tuberculosis. 2014;63:345-9.

24. Bari MR, Hiron MM, Zaman SM, Ganguly KC. Microbes responsible for acute exacerbation of COPD. Mymensingh Med J. 2010;19(4):576-85.

25. Wilson R. Bacteria, antibiotics and COPD. Eurpoean Respiratory Journal. 2001;995-1007.

26. Patel I, Seemungal T, Wilks M, Lloyd-Owen S, Donaldson G, Wedzicha J. Relationship between bacterial colonization and the frequency character and severity of COPD exacerbation. Thorax. 2002; 57(9):759-64.

27. Ram FS, Rodriguez-Roisin R, Granados-NavarreteA, GarciaAymerich J, Barnes NC. Antibiotics for exacerbations of chronic obstructive pulmonary disease. Cochrane Database Syst Rev. 2006;19(2):CD004403.

28. Patel A, Wilson R. Newer fluoroquinolones in the treatment of acute exacerbations of COPD. Int J Chron Obstruct Pulmon Dis. 2006;1(3):243-250. 